Mirtha Baina Villareal. Thomaz Marin Silva. Daiane Domingues Pereira. Jhonny Alvarez Lujan. Maria Del Carmen Saravia.

Correspondencia a:

Centro de investigación medicina UNITEPC. CIMU

Cochabamba - Bolivia

mirthabaina@unitepc-mail. com

thomaz_marin@unitepc-mail.com

daiane_domingues@unitepc-mail.com

jhonny_alvarez@unitepc-mail.com

mcarmen_saravia@unitepc-mail.com

Procedencia y arbitraje: No comisionado, sometido a arbitraje externo

Recibido para publicación: 1 de julio del 2018 Aceptado para publicación: 1 de septiembre 2018

Citar como:

$\mathrm{Re} \mathrm{Ci}$ Sa UNI

2018;5(2):23-27

\section{FRECUENCIA DEL CONSUMO DE DROGAS Y LA INFLUEN- CIA PARA CONSUMIRLAS EN ESTUDIANTES DE MEDICINA}

\section{FREQUENCY OF DRUG USE AND THE INFLUENCE TO CONSUME THEM IN MEDICINE STUDENTS}

\section{FREQUÊNCIA DO USO DE DROGAS E A INFLUÊNCIA DO CONSUMO EM ESTUDANTES DE MEDICI}

\section{Resumen.}

Se ha incrementado el consumo de drogas en determinados grupos sociales, entre ellos los estudiantes de medicina, el objetivo del presente estudio es, establecer la frecuencia de estudiantes que consumen drogas y la influencia para consumirlas en la carrera de medicina de la Universidad Técnica Cosmos UNITEPC. Cochabamba - Bolivia.

El tipo de estudio fue descriptivo, observacional y transversal, la población en estudio estuvo constituida por 428 estudiantes. Los resultados mostraron, consumo de marihuana $3 \%$, y cocaína $1.4 \%$. Y que el $23 \%$ fueron influenciados para iniciar a consumir drogas.

La Frecuencia del consumo de drogas ilícitas varia de una universidad a otra, como se puede ver en varios estudios, y los estudiantes de medicina son el grupo más influenciados para el consumo de drogas.

Palabras clave: Frecuencia, consumo, influencia, ilícita, drogas.

\section{Summary.}

Drug consumption has increased in certain social groups, including medical students, the objective of this study is to establish the frequency of students who use drugs and the influence to consume them in the medical career of the Technical University Cosmos UNITEPC . Cochabamba - Bolivia.

The type of study was descriptive, observational and transversal, the study population consisted of 428 students.

The results showed, $3 \%$ marijuana use, and $1.4 \%$ cocaine. And that $23 \%$ were influenced to start using drugs.

The frequency of illicit drug use varies from one university to another, as can be seen in several studies, and medical students are the most influenced group for drug use.

Keywords: Frequency, consumption, influence, illicit, drugs.

\section{Resumo.}

O aumento da quantidade de droga em determinados grupos sociais, entre os estudantes de medicina, o objetivo do presente estudo é estabelecer a frequência de estudantes que consomem drogas e a influência para o consumo destas no curso de medicina da Universidade Técnica Cosmos UNITEPC. Cocha- 
bamba - Bolívia.

O tipo de estudo é descritivo, observacional e transversal, a população em estudo é constituído por 428 estudiantes.

Resultados nos revelaram que, consumo de maconha 3\% e cocaína 1,4\%. E que em $23 \%$ destes foram influenciados para iniciar um consumo de drogas.

A frequência do consumo de drogas ilícitas varia de uma universalidade, como se pode ver em varios estudios, e os estudantes de medicina são o grupo mais influenciado para o consumo de drogas.

Palavras-chave: Frequencia, consumo, influencia, ilícita, drogas.

\section{INTRODUCCIÓN}

Sigue creciendo el consumo de drogas y es un problema de salud pública debido a sus efectos catastróficos en nuestra sociedad, en la actualidad mundial se observa un elevado uso de drogas ilícitas con una cifra de 230 millones de personas con edad entre 15 y 65 años de edad. En cuanto al consumo de tabaco millones de personas fuman, siendo que uno de cada diez adultos mayores de 30 años muere por consecuencia del uso crónico de esta droga. ${ }^{1}$

Una droga es una sustancia no producida por el organismo que tiene la propiedad de actuar sobre uno o más sistemas, produciendo cambios en su funcionamiento. Así, las drogas no son necesariamente nocivos, ya que pueden incluso proveer beneficios cuando se usan con precaución y por prescripción médica. Sin embargo, estas drogas, son capaces de alterar el funcionamiento del sistema nervioso central; son las denominados drogas psicotrópicas. ${ }^{2}$

Estas drogas pueden ser de origen natural o sintéticas y actúan en el cerebro, afectando procesos mentales, motores y emocionales que modifican la actividad psíquica y el comportamiento (excitando, deprimiendo y perturbando). Son sustancias que pueden causar dependencia y necesitan, por consiguiente, cuidado en su uso. ${ }^{3}$

Para entender mejor el fenómeno de las drogas, resulta elemental reconocer los factores de riesgo para su uso, así como los factores de protección. La dependencia a las drogas es un fenómeno complejo, siendo diversas las disciplinas del conocimiento científico necesario para su comprensión.

En la actualidad, hay un aumento sin precedentes en el uso de drogas por diversos grupos sociales. Uno de los que llama la atención de las autoridades es el de los estudiantes universitarios. Se enfrentan a situaciones que componen un proceso especial ya que muchos de ellos están en una fase de la vida que presenta una serie de conflictos tanto cognitivos como afectivos y al no tratarse adecuadamente, pueden salir de contro. ${ }^{4}$

Los efectos en el consumo de drogas en universitarios pueden reducir el rendimiento académico, y llevar al abandono social de los estudiantes y por consiguiente evita culminar con sus estudios. ${ }^{5}$

Las diferentes universidades son los centros adecuados para la realización de estudios relacionados con la salud de esta población universitaria con el fin de obtener datos de conocimientos y prácticas habituales de los jóvenes relacionados con este campo. 
El objetivo del presente estudio es establecer la frecuencia del consumo de drogas por estudiantes y la influencia para consumirlas en la carrera de medicina de la Universidad Técnica Cosmos UNITEPC. Cochabamba Bolivia.

\section{METODOLOGÍA}

El tipo de estudio fue descriptivo, observacional y transversal.

La población en estudio estuvo constituida por 428 estudiantes 170 hombres y 258 mujeres de la carrera de medicina de la Universidad Técnica Cosmos UNITEPC.

La recolección de datos se llevó a cabo en aula previamente coordinado con el docente de la asignatura, usando un cuestionario con preguntas cerradas. Para la tabulación y el análisis de datos se utilizó el programa Statistical Program Social Science (SPSS).

\section{RESULTADOS}

Durante el mes de febrero del 2017 se aplicaron encuestas a 428 estudiantes, los resultados muestran que el consumo de drogas en estudiantes de medicina es: Marihuana $13(3 \%)$, y cocaína $6(1.4)$. cuadro 1.

Cuadro 1.

Consumo de drogas en estudiantes de medicina.

\begin{tabular}{lcc}
\hline Droga & $\boldsymbol{N}^{\circ}$ & $\%$ \\
\hline Marihuana & 13 & 3.0 \\
Cocaína & 6 & 1.4 \\
\hline
\end{tabular}

La segunda pregunta fue, si en algún momento en la universidad habían sido influenciados a consumir drogas, los resultados mostraron que $328(77 \%)$ no fueron influenciados y 100 (23\%) respondieron que sí. Cuadro 2.

\section{Cuadro 2.}

Influencia al consumo de drogas en estudiantes de medicina.

\begin{tabular}{lcc}
\hline $\begin{array}{l}\text { Influencia a consumo de } \\
\text { drogas }\end{array}$ & $\mathbf{N}^{\circ}$ & $\%$ \\
\hline SI & 100 & 23 \\
NO & 328 & 77
\end{tabular}

\section{DISCUSIÓN}

Este estudio indica que el $3 \%$ de los estudiantes de medicina consumen marihuana y $1.4 \%$ cocaína, existen varios estudios en la región de Sudamérica, esta prevalencia del consumo de drogas ilícitas varia de una universidad a otra, como se puede ver en varios estudios realizados. Entre ellos un estudio realzado en Perú donde indican que el $16.7 \%$ consumen marihuana y $7,6 \%$ cocaína. $^{6}$

Bustamante indica que los resultados que se encontraron en una universidad de Lima fueron: el consumo de marihuana del $5,9 \%$, y $0,7 \%$ para la cocaína. ${ }^{7}$ Otro estudio acerca de las normas percibidas por los estudiantes universitarios y el uso de drogas, indica para marihuana $2,6 \%$ y $1 \%$ para la cocaína. ${ }^{8}$ 
El 23\% de los encuestados indicaron que en algún momento en la universidad fueron influenciados para consumir drogas.

Según estudios relacionados, los estudiantes universitarios son el grupo que más son influenciados para el consumo de drogas. ${ }^{9}$ Siendo la droga más consumida el alcohol, ya que se trata de un consumo extendido y aceptado por la sociedad. No obstante, más preocupante resulta la elevada incidencia que sugiere otros estudios, con el consumo de otras sustancias (marihuana y cocaína). ${ }^{10}$

El alcohol y el tabaco forman parte del estilo de vida ya que son elementos que se conciben como parte de la socialización, Aun cuando el excesivo consumo no se traduzca en casi ningún caso en alcoholismo, no por ello son de menor importancia las repercusiones sociales del consumo. ${ }^{11}$

Las drogas son sin duda un problema importante en nuestra sociedad, que conllevan dificultades en el desarrollo físico, psicológico, social y profesional. La edad de inicio disminuye cada año y cada vez están más accesibles para nuestra población de universitaria. $^{9}$

Gran parte de la sociedad boliviana no ven al alcohol y al tabaco como drogas que dañan a la sociedad, y son muy pocas las actividades sociales donde no esté el consumo de alcohol, además desde muy niños se socializa con el tema del consumo de bebidas alcohólicas.

Este estudio tiene limitantes, como indicar si después de ser influenciados los estudiantes experimentaron con drogas y el tipo de droga, lícita o ilícita.

No está entre las preocupaciones de nuestra sociedad el tratar el poner un el alto al consumo de sustancias en los jóvenes universitarios, quienes son los futuros profesionales de la salud, y que difícilmente podrán realizar una adecuada labor de transmisión de hábitos saludables si ellos mismos mantienen una actitud positiva hacia el consumo de sustancias. Resulta pues, urgente, la intervención para solucionar este problema universitario.

\section{BIBLIOGRAFÍA}

1. United Nations Office on Drugs and Crime (UNODC). Global study on homicide: trends, context, data. Vienna (AT): UNODC; 2011 [acesso 2011 Jul 15]. Disponível em: http://www.unodc.org/documents/data-and-analysis/statistics/Homicide/ Globa_study_on_homicide_2011_web.pdf

2. Brasil. Presidência da República. Secretaria Nacional de Políticas sobre Drogas. I Levantamento Nacional sobre o uso de álcool, tabaco e outras drogas entre universitários das 27 capitais brasileiras. Brasília (DF): SENAD; 2010.

3. Zeferino MT, Fermo VC. Prevenção ao uso/abuso de drogas. In: PROENF. Saúde do Adulto v.2. Porto Alegre (RS): Artmed/Panamericana; 2012. p. 9-42.

4. Organization of American States, Inter-American Drug Abuse Control. Multilateral Evaluation Mechanism. Governmental Expert Group: Brazil. Assessment of Progress in Drug Control 2007-2009. Washington (DC): CICAD/OAS; 2010.

5. Intra MV, Roales-Nieto JG, Mo- reno San Pedro E. Cambio en las conductas de riesgo y salud em estudiantes universitarios ar- gentinos a lo largo del periodo educativo. International Jour- nal of Psychology and Psycho- logical Therapy. 2011 
(11-1): $139-47$.

6. Zárate $M$, Zavaleta A, Danjoy $D$, et al. Prácticas de consumo de tabaco y otras drogas en estudiantes de ciencias de la salud de una universidad privada de Lima, Perú. Invest.educ.enferm. 2006;24(2):72-81.

7. Bustamante I, Strike C, Brands B, Cunningham J, Wright MGM. Normas percibidas por estudiantes peruanos acerca de sus pares y el uso de drogas. Rev Latino-am Enferma- gem. 009;17:858-64.

8. Chanamé E, Cunningham J, Brands B, Strike C, Wright MGM. Normas percibidas por los estudiantes universitarios de enfermería acerca de sus pares y el uso de drogas en Lima, Perú. Rev Latino-am Enferma- gem. 2009;17:865-70.

9. Ministerio de Sanidad, Servicios Sociales e Igualdad (2013). Encuesta Domiciliaria sobre Alcohol y Drogas en España (EDADES). Recuperado de http://www.pnsd. msssi.gob.es/profesionales/sistemasInformacion/sistemalnformacion/encuestas. $\mathrm{htm}$

10.Pascual-Pastor, F., Guardia, J., Pereiro, C. y Bobes, J. (2013.) Alcoholismo: Guía de intervención en el trastorno por consumo de alcohol. Guías clínicas Sociodrogalcohol basadas en la evidencia científica ( $3^{a}$ Ed.). Esplugues de Llobregat, España: SANED.

11. Barrett, S., Darredeau, C. y Pihl, R. (2006). Patterns of simultaneous poly-substance use in drug using university students [Los patrones de policonsumo de drogas en los estudiantes universitarios]. Human Psychopharmacology, 21, 255-263. http:// dx.doi.org/10.1002/hup.766 